\title{
essai de chargement d'une fondation superficielle sur une pente d'éboulis
}

\author{
load test of a shallow foundation on a scree-covered slope
}

\author{
J.P. ASTE \\ Chef de l'atelier Risques Naturels et Aménagement en Montagne du B.R.G.M. à Lyon* \\ Y. GUERPILLON \\ Ingénieur, chef de la division Laboratoire, Scetauroute Annecy**
}

\section{Résumé}

Au niveau des Neyrolles, près de Nantua, le tracé de l'autoroute A40 MâconGenève se développe en viaduc sur une pente d'éboulis. Les fondations du viaduc se font par l'intermédiaire de puits en gros diamètre poussés au substratum calcaire sous-jacent. Une variante a cependant été proposée sous forme de semelle inclinée associée à des tirants d'ancrage. Cette variante a fait l'objet d'un essai de simulation in situ, avec une semelle réduite au quart de celle du projet et chargée par l'intermédiaire de tirants verticaux.

L'essai de chargement a été contrôlé au moyen de nombreux appareils d'auscultation aux caractéristiques complémentaires, qui ont permis un suivi extrêmement précis du champ de déformations développé à la surface du sol et sous l'ouvrage.

L'interprétation des résultats permet de penser que des solutions superficielles peuvent devenir concurrentielles par rapport aux solutions traditionnelles adoptées jusqu'à maintenant.

\footnotetext{
Abstract

At the level of Les Neyrolles, near Nantua, the motorway A40 (Mâcon-Genève), uses a viaduct on a scree-covered slope. The foundations of the viaduct are big diameter piers anchored in the underlying limestone. However, an another design was proposed: an inclined shallow foundation with anchors. This last design was tested in the field, using a footing four times smaller than the project size, and loaded using vertical anchors.

The load test was controlled using various monitoring devices giving complementary informations. They made possible to follow very accurately the spreading displacements at the ground surface and under the footing.

Interpretation of the output shows that shallow foundations may be competitive when compared with the more traditional designs used untill now.
}

\footnotetext{
* Services géologiques Rhône-Alpes, BP 6083 - 69604 Villeur-

banne.

* Division Laboratoire, BP 122 - 01130 Nantua.
} 


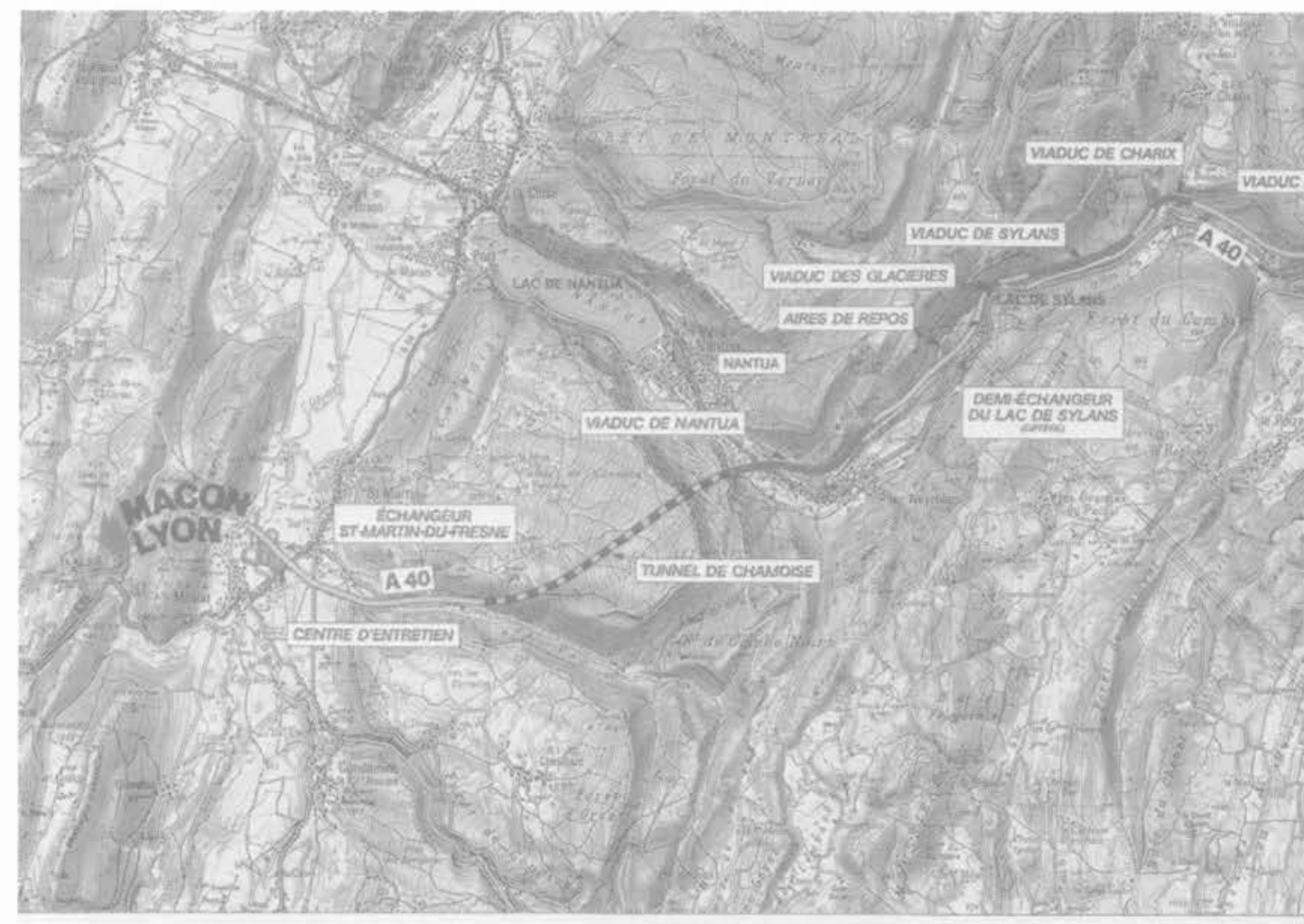

A 40 UAISON AUTOROUTIĖRE TUNNEL DU MONT BLA FRANCHISSEMENT DU JURA MÉRIDIONAL PAR LA CLUSE NA CARACTERISTOUES GEOMETRIOUES

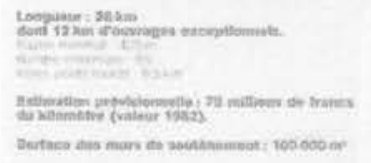

OUVRAGES D'ART EXCEPTIONNELS

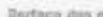
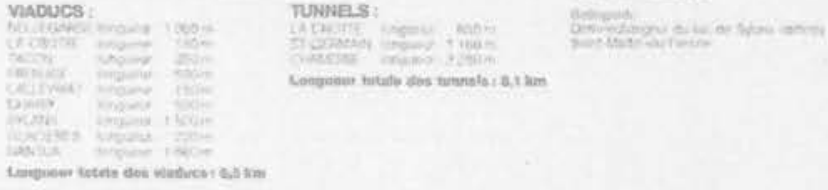

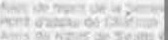

1.

\section{CONTRAINTES GÉOTECHNIQUES DU SITE DE L'AUTOROUTE A40}

L'Autouroute A40 Mâcon-Genève assure la liaison entre deux axes autoroutiers qui traversent l'Europe du Nord au Sud, soit par la vallée du Rhône, soit par l'Allemagne, la Suisse et l'Italie.

Son intérêt économique est évident, c'est le dernier maillon de la liaison Paris-Rome par le tunnel sous le Mont Blanc. Actuellement trois mille poids lourds par jour empruntent la Route Nationale entre Pont d'Ain et Bellegarde.

Mais le problème est difficile, il faut franchir le Jura. Le Jura est formé de chaînons parallèles d'orientation Nord-Sud. Il est entrecoupé dans le sens Est-Ouest seulement par quelques cluses qui furent empruntées très tôt par les axes de communications.

\subsection{Cadre général}

L'autoroute $\mathrm{A} 40$ a choisi la cluse de Nantua (fig. 1). Elle a une morphologie bien typique: fond de vallée étroite, versants souvent raides et dominés par des falaises. Comme il n'y avait pas de place en fond de vallée, l'autoroute a dû être implantée à flancs de côteau.

Les versants ont souvent une inclinaison variable liée à la nature du sous-sol:

- fortes pentes supérieures à $40^{\circ}$ avec ie rocher sous une faible couverture;

- pente de 30 à $40^{\circ}$, généralement des éboulis calcaires;

- des pentes plus douces, 20 à $25^{\circ}$ pour les dépôts glaciaires:

- des pentes faibles de 10 à $15^{\circ}$ dans les combes argileuses. 

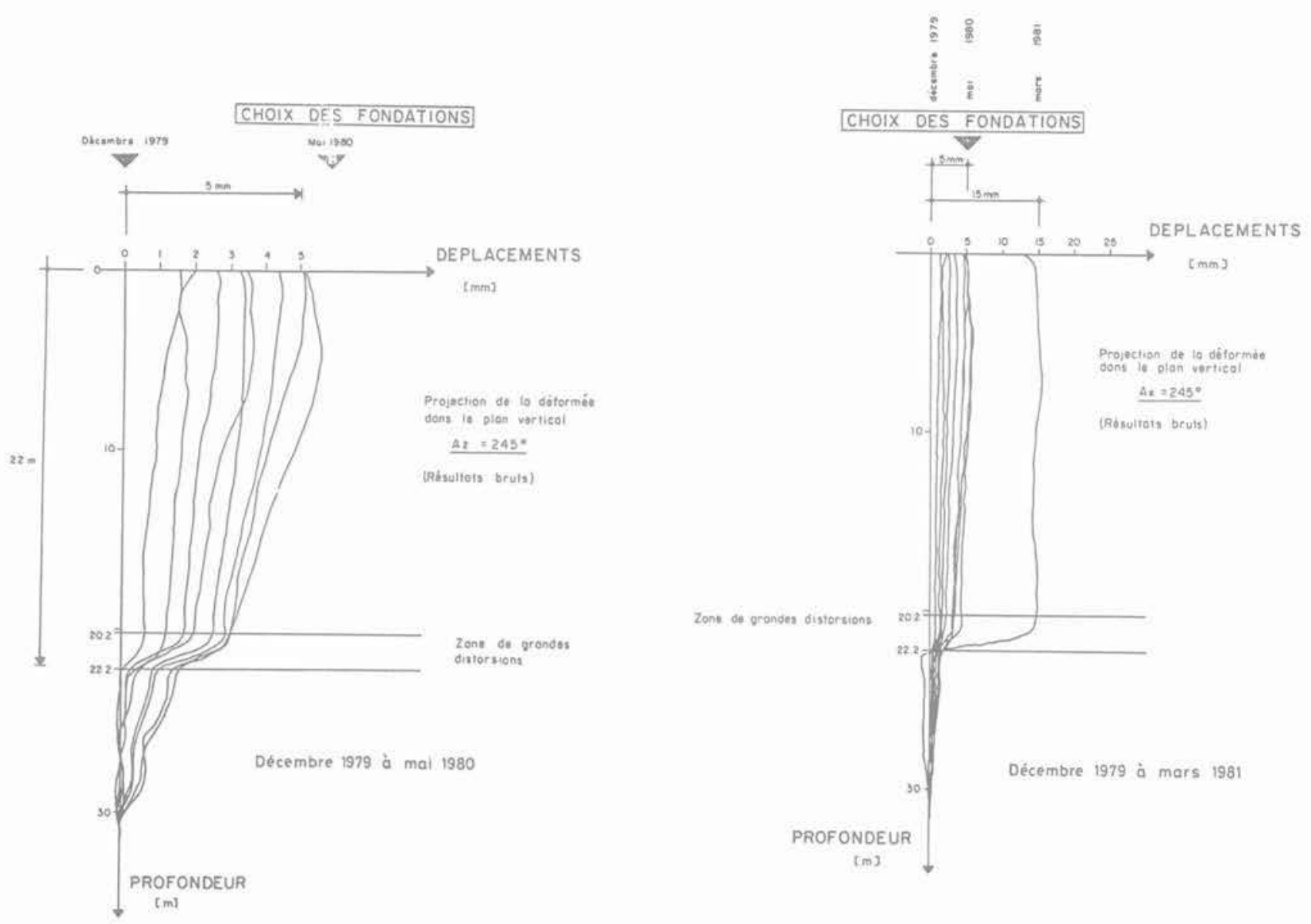

Fig. 2. - Relevés inclinométriques.

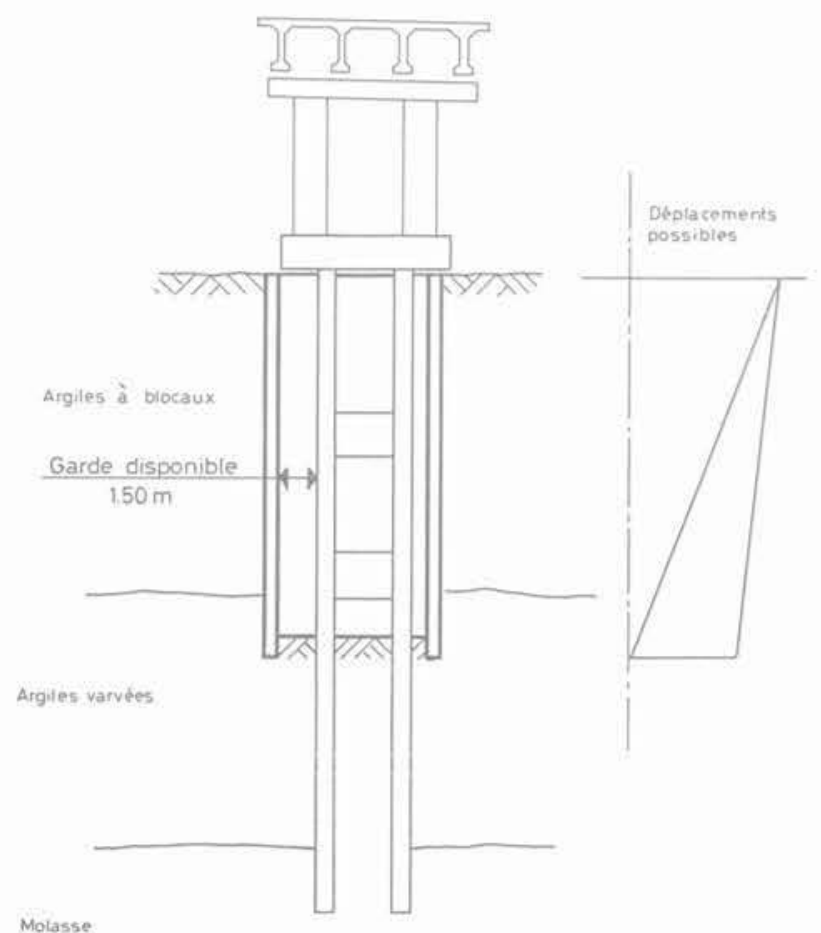

Fig. 3. - Barettes protégées par caissons béton. décalées et dénivelées afin de limiter au maximum la hauteur des soutènements (fig. 4).

Pour des pentes supérieures à $35^{\circ}$, une solution terrassement n'est plus possible même en utilisant au maximum la technique des murs ancrés. En effet, la largeur nécessaire pour la plateforme autoroutière impose une hauteur de mur de remblai ou de déblai incompatible avec la stabilité des pentes. La solution viaduc est alors retenue. Il s'agit d'ouvrages de grandes longueurs à faible hauteur de pile dont la travure économique est de 50 à $60 \mathrm{~m}$. Il reste à résoudre le problème des fondations.

\subsection{Pente déboulis et type de fondations}

Le substratum rocheux se situe généralement à une profondeur de 5 à $25 \mathrm{~m}$.

Les terrains de couvertures sont essentiellement constitués d'éboulis. Ils proviennent de l'altération du substratum rocheux et sont formés de débris et blocs calcaires de différentes dimensions pouvant aller jusqu'au $\mathrm{m}^{3}$, avec ou non une matrice silteuse.

On peut distinguer trois classes principales pouvant former des couches parallèles à la pente: éboulis à gros blocs, éboulis à matrice silteuse, éboulis aérés. Les 


\section{Section Chatillon-St.Martin}

\section{Murs de soutènement}

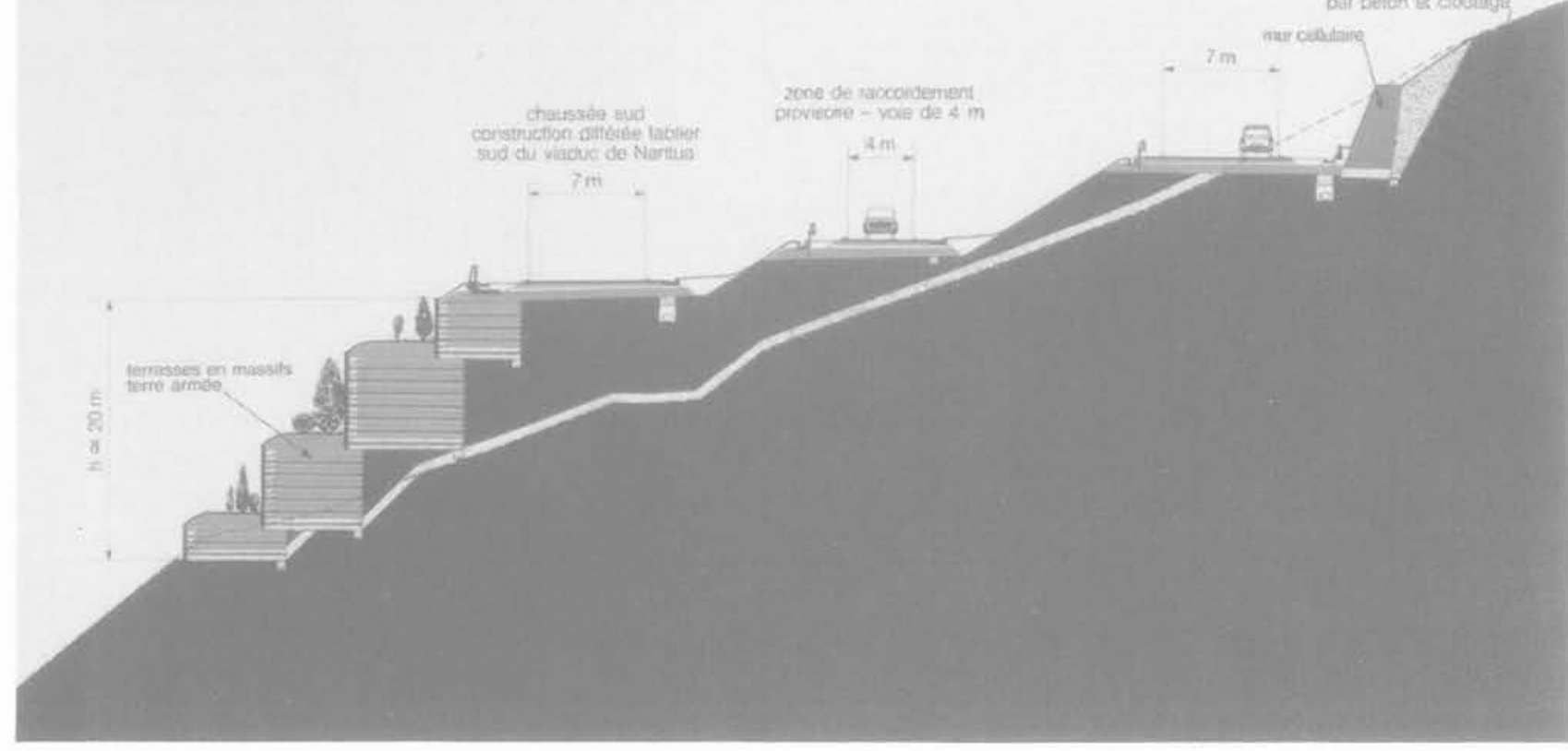

\section{Section Chatillon-St.Martin}

\section{Murs de soutènement}

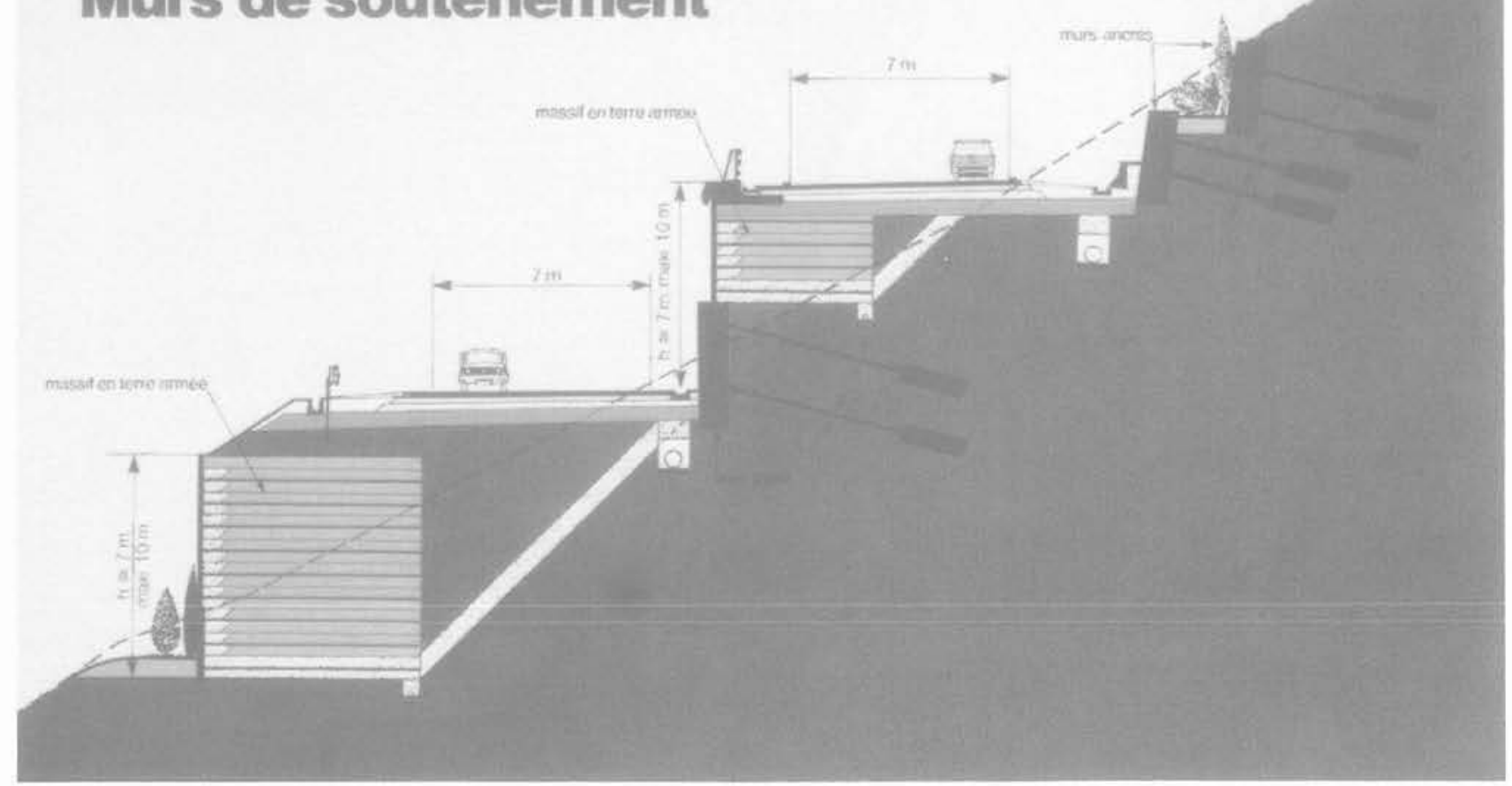

Fig. 4. - Terrassements à flanc de versant. 
éboulis aérés sont les plus critiques. lis sont formés de graviers et galets avec vides entre eux et correspondent presque exclusivement à une seule classe granulométrique. Des dépôts glaciaires sont généralement rencontrés en faible épaisseur au toit du substratum rocheux.

La détermination des caractéristiques mécaniques de tels sols est difficile, aussi avons nous dû procéder par recoupement entre différentes corrélations issues de plusieurs types d'essais soit sur échantillons remaniés, soit en faisant appel pour une large part aux essais in situ.

Par exemple, le viaduc des Neyrolles d'une longueur d'environ $800 \mathrm{~m}$ est situé au pied du Mont Cornet à flanc de coteau d'un versant d'éboulis.

Dans la zone de l'essai de chargement entre les piles $\mathrm{P} 21$ et P 22, on peut considérer être en présence d'éboulis silteux et d'éboulis aérés d'angle de frottement $\varphi^{\prime}=35^{\circ}$ et de cohésion $c^{\prime}=2$ à $10 \mathrm{kpa}$. Les modules pressiométriques et pressions limites sont très variables respectivement 3 à $50 \mathrm{Mpa}$ et 0,5 à $4 \mathrm{Mpa}$.

Toutes les observations et mesures que nous avons pu faire ont confirmé l'état de stabilité du versant. Il n'y a pas de signes de mouvements actifs. L'auscultation des versants a consisté en:

- mesures topographiques de surface entre 1976 et 1979 à la précision de $2 \mathrm{~cm}$;

- restitution photogrammétrique de deux séries de photos aériennes de 1955 et 1979 à la précision du mètre;

- mesures inclinométriques dans les zones les plus sensibles depuis 1979.

Mais compte tenu des caractéristiques mécantiques des éboulis, de leur mode de formation et de la forte pente transversale du TN, les coefficients de sécurité à la stabilité générale ne peuvent guère être supérieurs à l'unité.

Une solution simple de fondation de pile de viaduc dans de tels versants peut être constituée par un puits unique de diamètre 4 à 6 mètres descendant les charges au substratum (fig. 5). Ce type de fondation peut être exécuté par des moyens légers qui perturbent au minimum l'équilibre des pentes.

Mais il semble un peu archaïque et on peut chercher à faire participer les terrains de couverture dont les caractéristiques mécaniques sont élevées.

\subsection{Fondations utilisant les terrains de couverture}

Dans cette perspective, nous avons étudié une solution de fondations sur micropieux ou sur semelle renforcée par tirants d'ancrage.

La solution micropieux est intéressante. D'après l'expérience italienne, on peut envisager un véritable massif constitué de pieux inclinés qui encagent le terrain. Suivant ce principe, il faudrait environ quarante à cinquante pieux de $450 \mathrm{kN}$ par appui à la maille de $1 \times 1 \mathrm{~m}$ (fig. 6).

La solution semelle renforcée a été proposée par l'entreprise Bouygues. Il s'agit d'une semelle inclinée suivant la pente du versant associée à des tirants d'ancrage inclinés de $30^{\circ}$ sous l'horizontale conduisant à rendre les efforts apportés par l'ouvrage normaux au versant (fig. 7).

Ces deux types de fondations posent des problèmes de dimensionnement. Aussi, nous avons réalisé des essais de chargement in situ.

Les questions que pose une fondation sur semelles sont de deux ordres:

- quels seront les tassements de sols granulaires simplement déposés sur les pentes donc de faible densité et renfermant de nombreux vides;

- quelle est l'influence d'une telle fondation sur la stabilité générale du versant.

L'objectif d'un essai de chargement était donc par la mesure des déplacements en profondeur et autour de la semelle, d'apprécier la répartition des efforts d'une semelle sur un sol incliné, répartition encore très mal connue.

Cet essai a été réalisé sur le versant du viaduc des Neyrolles actuellement en cours de construction.

\section{PRINCIPE DE L'ESSAI ET DISPOSITIF EXPÉRIMENTAL}

La connaissance des éboulis du site était basée essentiellement sur les études générales du tracé.

Au droit du site retenu pour l'expérimentation, on disposait des résultats d'un sondage carotté NP 21 $\mathrm{SC2}$, et de deux sondages pressiométriques NP 20 WD1 - NP 21 WD1, ainsi que d'un puits de reconnaissance P $15-12$, et d'un profil en sismique réfraction. Sur le profil 21 , à une trentaine de mètres du site, nous avons fait réaliser par la Société Intrafor, deux puits expérimentaux de grand diamètre $(6 \mathrm{~m})$.

Avec ces résultats, il était difficile de faire une estimation précise des performances à attendre de la semelle en matière de force portante et de tassement. Mais l'attention du projecteur se portait d'abord sur la stabilité globale des éboulis, que l'on jugeait précaire et qui semblait compromettre la faisabilité d'une solution sur semelle superficielle.

Une approche théorique du comportement de la fondation superficielle sur la base des résultats d'essais pressiométriques, laissait prévoir des tassements d'ordre multicentimétrique à décimétrique pour les charges envisagées.

Une autre approche, inspirée des méthodes classiques relatives aux fondations soumises à des charges inclinées a également été réalisée. Avec les valeurs d'angle 

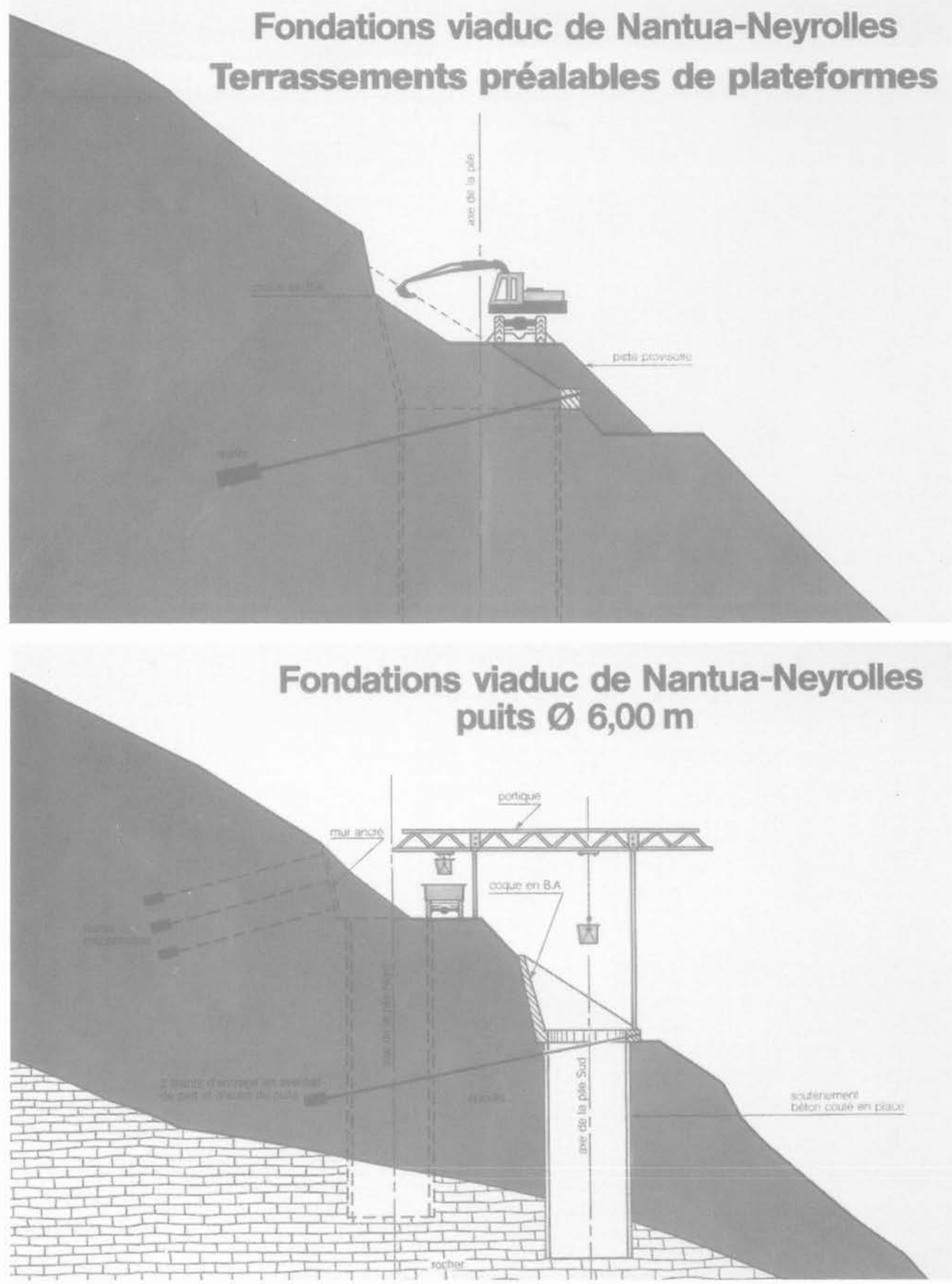

Fig. 5. - Fondations des viaducs. 


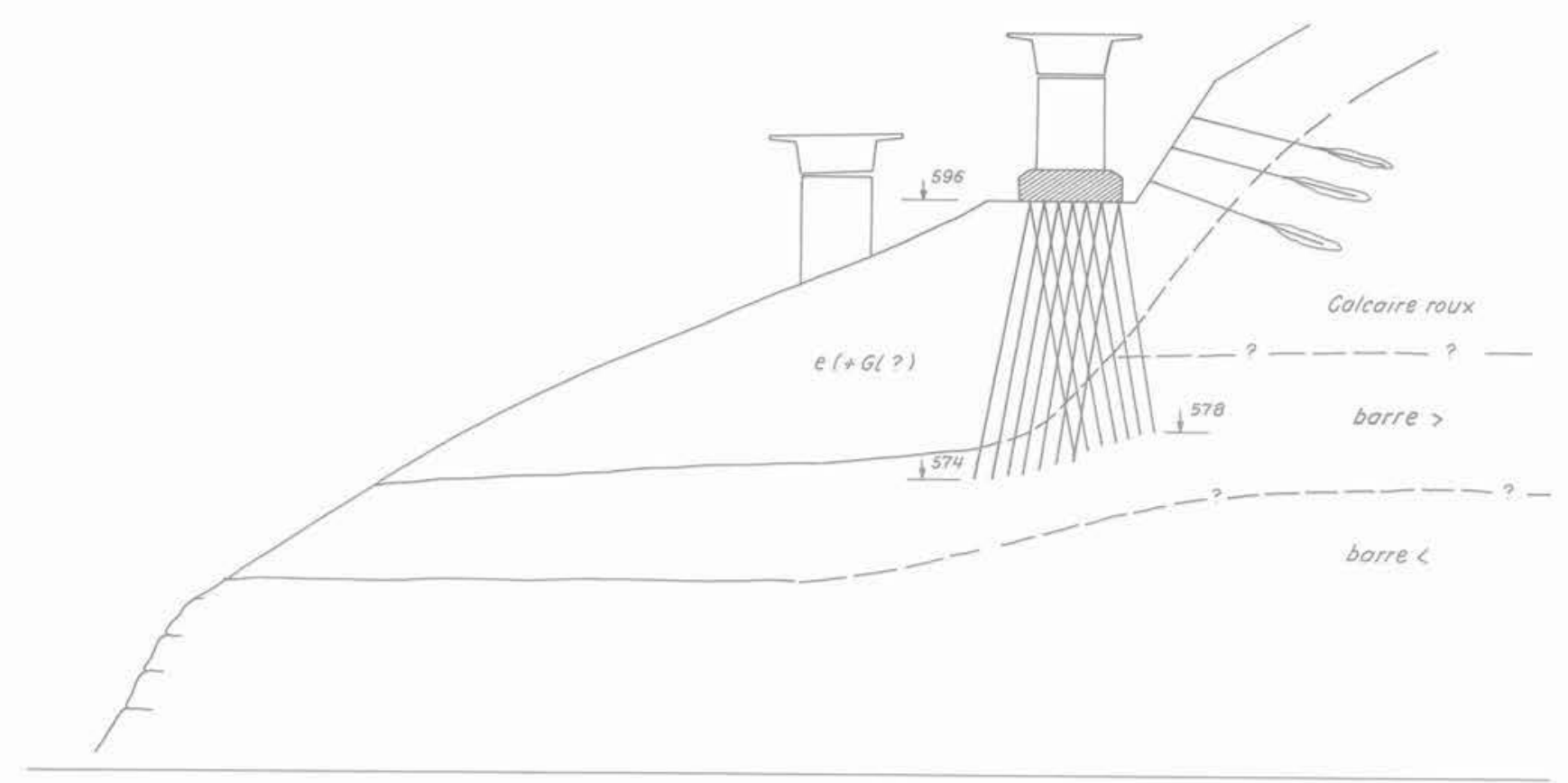

Fig. 6. - Fondations sur micropieux

(Adaptation au viaduc des Neyrolles).

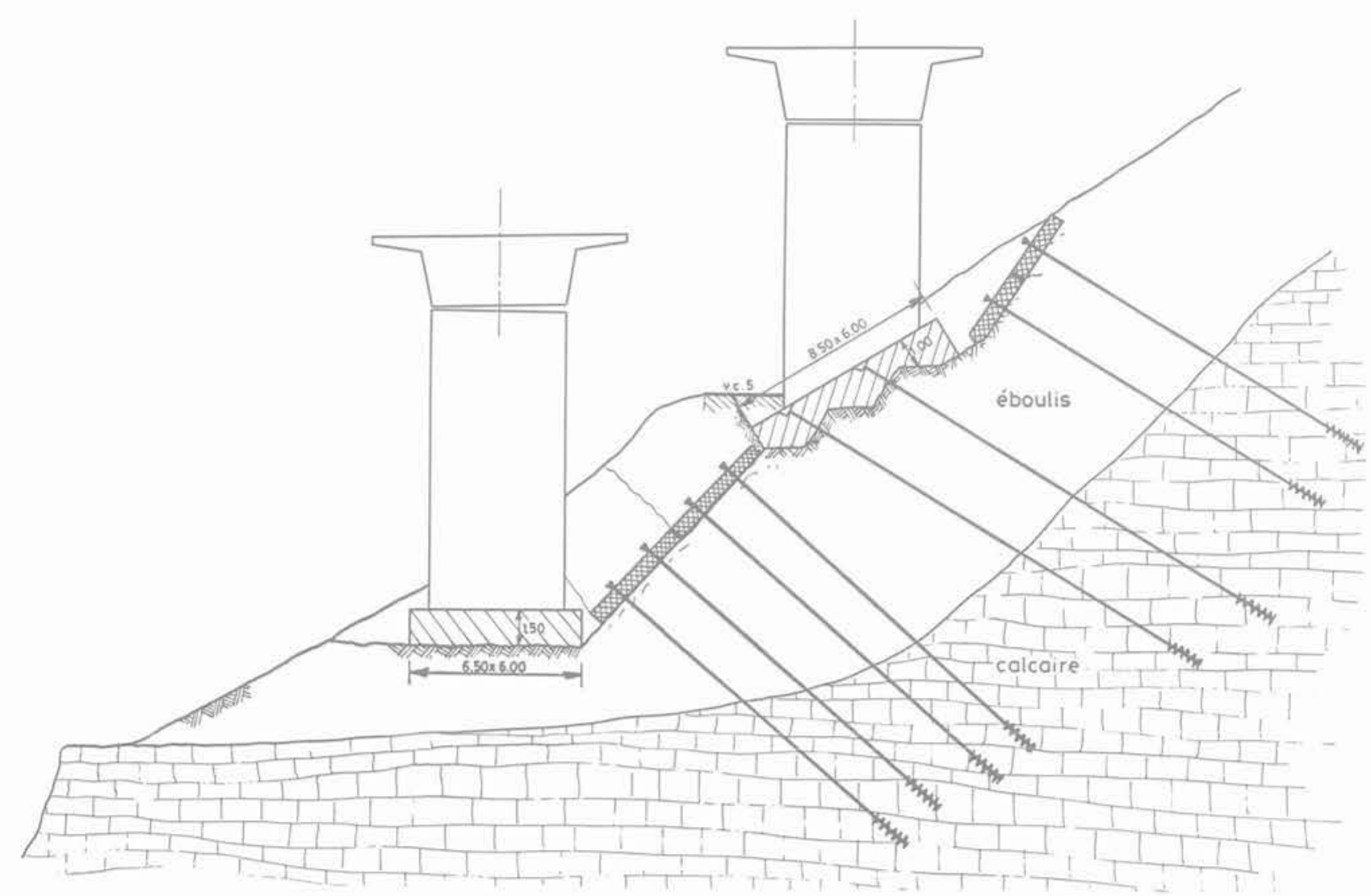

Fig. 7. - Piles 22 Neyrolles. Fondation sur semelle. 
de frottement envisagées, la force portante apparaissait limite pour une sollicitation verticale, plus confortable en introduisant une composante inclinée rentrant dans le massif par le biais de tirants.

On a étudié également la stabilité d'ensemble du versant, avec simulation d'une surcharge importante verticale.

Pour $\varnothing=35^{\circ}$, le coefficient de sécurité reste très légèrement supérieur à 1 pour une surcharge verticale et des surfaces de glissement circulaires de rayon faible,

Il devient très nettement inférieur à 1 pour de grands cercles, de glissement sauf à mobiliser une cohésion importante.

En fait, la coupe géologique interdit le développement de tels cercles de glissement (rocher à profondeur par trop importante).

Enfin, une simulation préalable du comportement du versant par la méthode des éléments finis a été menée. Elle mériterait d'être beaucoup plus approfondie grâce aux excellents résultats disponibles en matière de déformations et sollicitations.

\subsection{Mise en ceuvre de la sollicitation}

La semelle de fondation a été réalisée immédiatement à l'amont d'une piste de reconnaissance taillée dans la couverture d'éboulis à peu près deux ans auparavant. Cela a nécessité l'ouverture préalable d'une excavation supplémentaire dans le talus amont de la piste, excavation dont l'emprise a été limitée pour réduire le risque d'une déstabilisation superficielle du talus amont. Pour réduire encore davantage ce risque, on a construit immédiatement après l'excavation, un mur de soutènement de ce talus amont, ancré par quatre tirants de 25 tonnes.

La forme et les dimensions de la semelle sont indiquées sur la figure 8 . Son ferraillage a été conçu pour qu'elle puisse être considérée comme rigide sous les efforts très importants qu'elle devait subir, avec toutefois les réservations nécessaires pour la mise en cuvre des tirants de chargement et des forages d'auscultation.

On devait, sur cette semelle, simuler l'effet d'une charge verticale d'environ $1 \mathrm{O}$ MN. Cela a été fait au moyen de quatre tirants verticaux de 2,5 MN largement ancrés au rocher sous jacent pour éviter tout phénomène parasite au niveau du scellement.

Les vérins ont été mis en charge progressivement avec un dispositif permettant d'agir sélectivement sur chacun d'eux afin d'éviter toute distorsion dans les efforts appliqués et tout basculement intempestif de la semelle.

Une telle charge, verticale, donc oblique par rapport à la surface de la semelle, avait, pour la stabilité au poinçonnement comme pour la stabilité au glissement sous la semelle ou celle au glissement d'ensemble, une direction défavorable.

On a donc cherché à diminuer la composante tangentielle, c'est-â-dire à ramener la sollicitation plus près de la normale au massif en introduisant un effort de $2 \mathrm{MN}$ incliné à $30^{\circ}$ sur l'horizontale par l'intermédiaire de deux tirants supplémentaires.

Une fois l'ensemble de ces tirants mis en place, il a été décidé de mettre en ceuvre la procédure de chargement illustrée sur le schéma détaillé sur la figure 9.

\subsection{Mise en ceuvre de l'auscultation}

\subsubsection{Mise en tension et contrôle des tensions des tirants}

Compte tenu de la bonne qualité, a prion, du calcaire formant le substratum, on pouvait espérer éviter tout fluage des scellements.

Toutefois, les longueurs de scellement ont été largement dimensionnées pour s'affranchir de ce problème.

Pour les quatre tirants verticaux, la Société Intrafor a mis en ceuvre un dispositif de mise en charge permettant une mise en charge simultanée des quatre tirants et un réglage individuel sur chaque tirant. $\mathrm{Ce}$ dispositif évite un basculement de la semelle.

Ces quatre titrants ont été équipés de cales dynamométriques de contrôle de la tension type GLOTZL K 200.

Les deux tirants iclinés T1 et T2 assurant l'ancrage de la semelle ont été tendus à $1 \mathrm{MN}$ chacun en début d'essai.

Leur tension a été contrôlée pendant la première partie de l'essai à l'aide de deux cales dynamométriques type GLOTZL K 100.

\subsubsection{Contrôle des déplacements de la semelle}

La semelle d'essai en béton armé était dimensionnée et ferraillée pour pouvoir se déplacer en corps rigide. Le principe du contrôle du déplacement de la semelle a consisté en la détermination au cours de l'essai des coordonnées de trois points de la semelle par rapport à un repère fixe de référence $\mathrm{XYZ}$.

Les déplacements de la semelle dans le plan XY (Y étant l'axe longitudinal, sens de la pente, et $\mathrm{X}$ le sens transversal) ont été suivis à l'aide de six bases de mesures Distomatic.

- trois dans le sens longitudinal, chacune composée d'un plot fixé sur un des massifs de la semelle et d'un autre plot ancré à l'amont au-dessus du mur de soutènement:

- trois dans le sens transversal, chacune composée d'un plot fixé sur des massifs de la semelle et d'un autre plot ancré à une quinzaine de mètres de la semelle. 


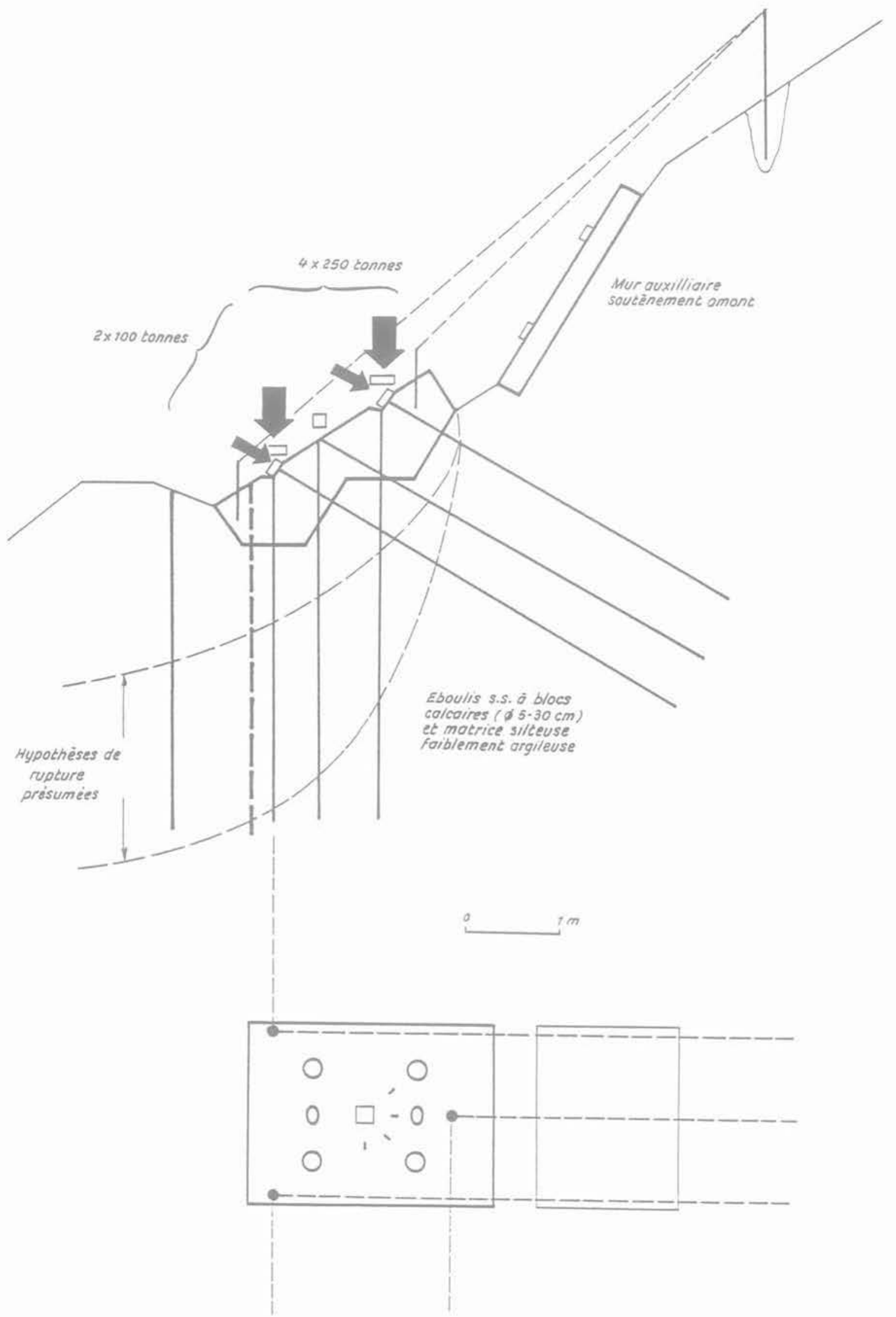

Fig. 8. - Plan et coupe. 

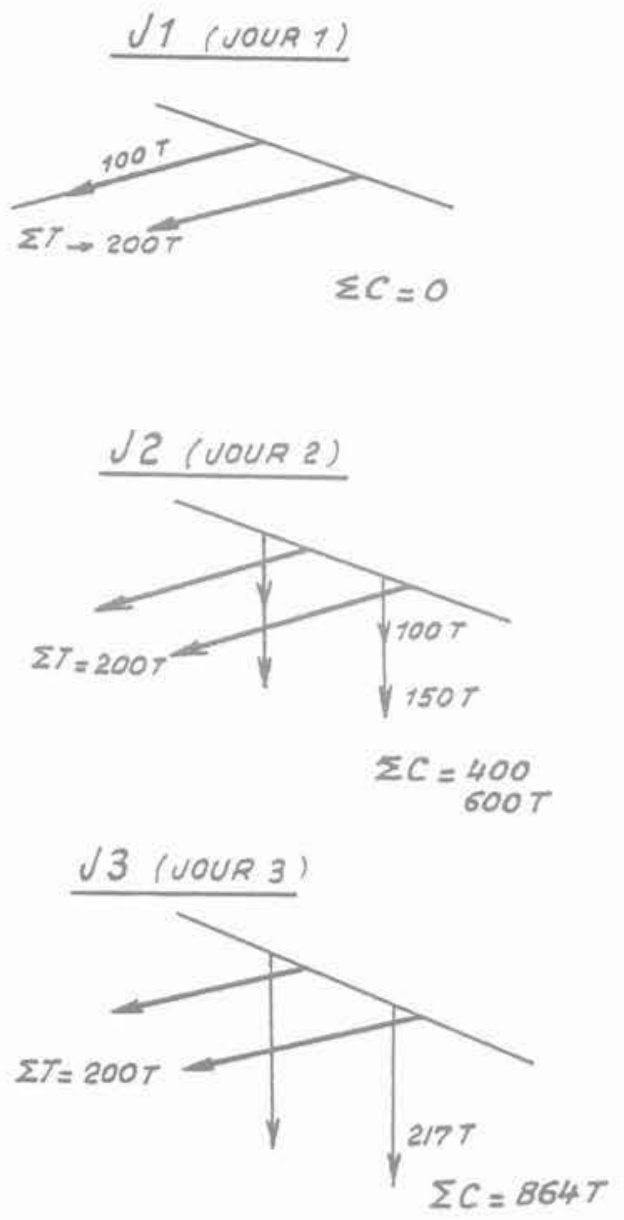

$\sqrt{4}(100 R 4)$
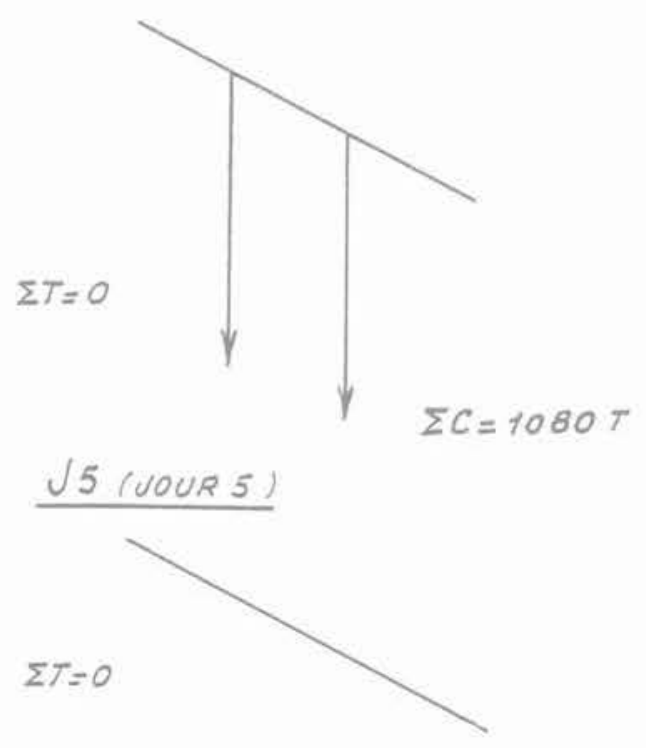

$$
\Sigma C=0
$$

Fig. 9. - Mise en ceuvre de la sollicitation.

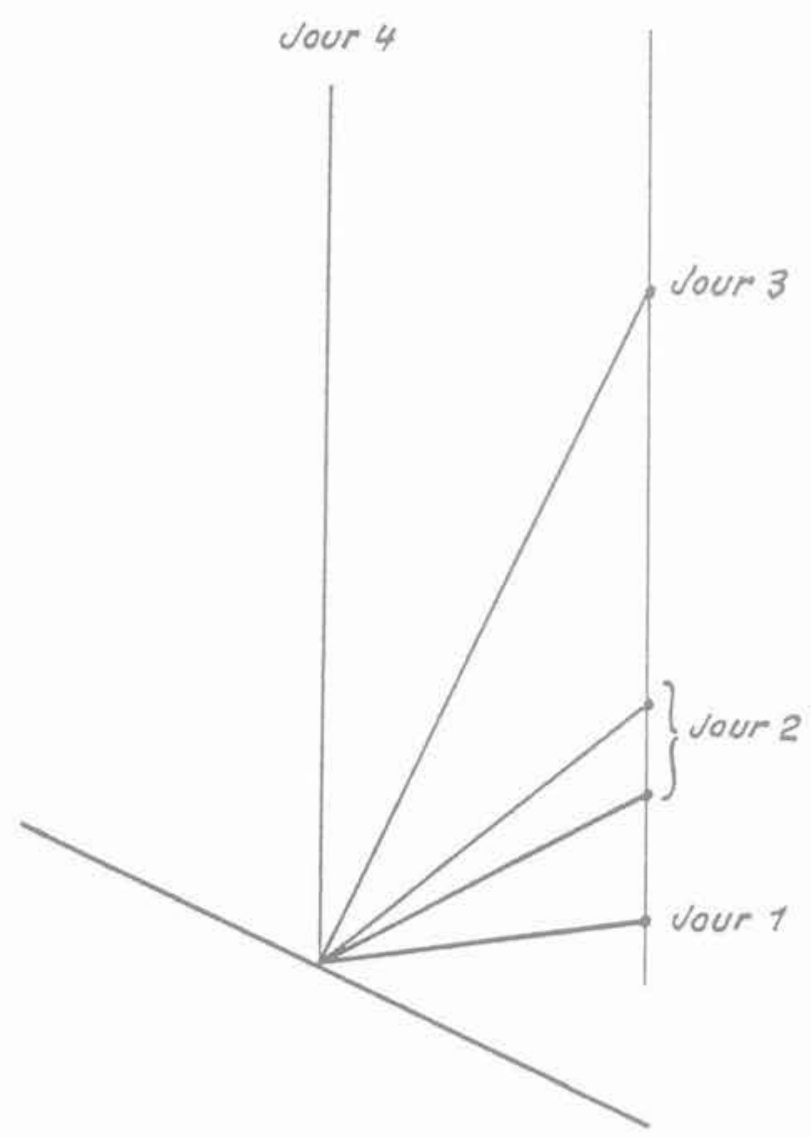


Dans le sens longitudinal, les trois plots de référence ont été fixés sur des profilés métalliques de $2,50 \mathrm{~m}$ de long encastrés de $1,00 \mathrm{~m}$ dans le sol et scellés à la base par du béton, à l'amont de la semelle au-dessus du mur de soutènement.

Dans le sens transuersal, on a fixé les plots de référence également sur des profilés métalliques scellés dans des massifs de béton disposés à une quinzaine de mètres de part et d'autre de la semelle.

Les déplacements verticaux de la semelle ont été suivis par deux types d'appareils différents:

1. au moyen d'un Nivomatic;

2. au moyen de relevés topographiques de précision.

Le Nivomatic n'est pas rigoureusement au centre de gravité des points de mesure topographique.

Le fonctionnement du. Nivomatic reposant sur le principe des vases communiquants, on a fixé le «plot mobilen approximativement au centre de la semelle sur un plot Distomatic scellé dans la semelle et le "plot de référence sur un des massifs servant de référence aux bases Distomatic.

Les mesures topographiques ont été faites avec un niveau optique Wild type NA2 muni d'un micromètre permettant de lire le $1 / 10^{e}$ de $\mathrm{mm}$ et d'apprécier le $1 / 100^{e}$ de $\mathrm{mm}$. On a également utilisé une mire Invar GPL.

On a enfin scellé sur la semelle quatre nivelles dans les quatre directions principales (les deux axes et les deux diagonales) à l'aide de ciment prompt en vue de déceler une éventuelle rotation de la semelle.

\subsubsection{Mesure des dêformations du sol}

Les déformations du sol ont été contrôlées à l'aide de deux appareillages distincts:

- deux sondages équipés d'Extensofors pour le contrôle des tassements,

- deux sondages équipés de tubes inclinométriques pour la surveillance des déplacements horizontaux et la détection d'éventuelles surfaces de rupture.

Les deux sondages inclinométriques ont atteint $15 \mathrm{~m}$ de profondeur.

Le premier est implanté dans l'axe de la semelle, 0,50 $\mathrm{m}$ à l'aval de celle-ci, le second latéralement à $1,50 \mathrm{~m}$.

Le sondage équipé de l'Extensofor E1, implanté au centre de la semelle et incliné à $30^{\circ}$ par rapport à l'horizontale, a été poursuivi jusqu'à $17 \mathrm{~m}$. Le calcaire a été atteint à $14 \mathrm{~m}$.

Le sondage équipé de l'Extensofor E2 vertical et implanté sur la même ligne transversale que E1, a atteint $14 \mathrm{~m}$, le calcaire a été atteint à $10 \mathrm{~m}$.

A l'issue de cette présentation sommaire des matériels utilisés, il convient de souligner l'origine de ces matériels:
- la nivelle est fabriquée et distribuée par le LRPC de Toulouse;

- les Distomatic, Nivomatic, Extensofor, par la Société Télémac;

- l'inclinomètre est un MK III de Soil Instruments;

- le boitier de saisie et de traitement des mesures inclinométriques est un prototype du BRGM Lyon.

\section{RÉSULTATS OBTENUS ET INTERPRÉTATION}

\subsection{Détail des rêsultats présentés}

Les résultats détaillés sont regroupés de la façon suivante:

- Déplacements superficiels, parallèlement au versant, mesures au Distomatic en fonction de la charge (fig. 10).

- Déplacements superficiels verticaux, mesures au Nivomatic et en topographie en fonction de la charge.

- Déplacements profonds, mesures en extensomètre en fonction de la charge (fig. 11).

- Mesures inclinométriques.

- Courbes effort-déformation (normales et tangentielles).

- Mesures effectuées sur les nivelles.

\subsection{Relation effort déplacement en surface}

\subsubsection{Diverses représentations}

Cette relation ne peut s'exprimer d'une façon simple puisque l'effort est variable non seulement en grandeur mais aussi en inclinaison.

En admettant que les déplacements latéraux $x$ restent négligeables, on peut tracer, dans le plan vertical de symétrie de la semelle son vecteur déplacement total $(\Delta Y+\Delta Z)$ en fonction du vecteur force $F$.

On observe:

- en cours de la mise en tension des tirants inclinés $\left(F_{1}\right.$ à $\left.F_{4}\right)$ que le déplacement est essentiellement un glissement vers l'amont,

- ensuite de $F_{5}$ à $F_{14}$ avec l'accroissement de l'effort vertical, le déplacement se fait vers l'aval et le bas. Il faut arriver à une charge verticale de $6 \mathrm{MN}$ pour rattraper le glissement amont (pour repasser par la verticale),

- à partir du moment où se fait le rechargement sous seul effort vertical (tirants inclinés détendus), les déplacements deviennent plus importants et leur composante vers l'aval est de plus en plus marquée.

Pour se rapprocher des courbes effort déplacements classiques, on a tracé dans un système d'axes ortho- 

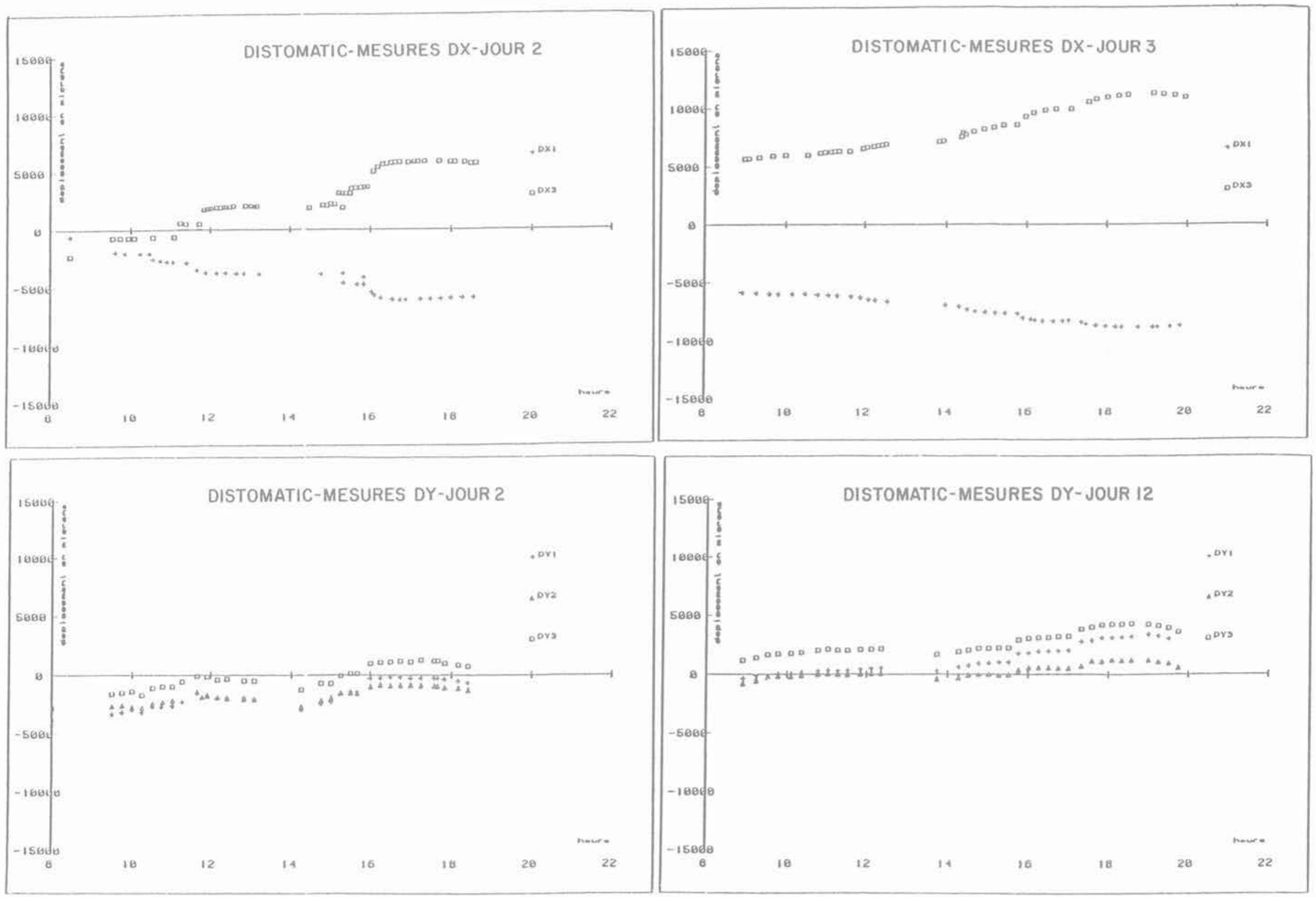

Fig. 10. - Semelle d'essai des Neyrolles. 


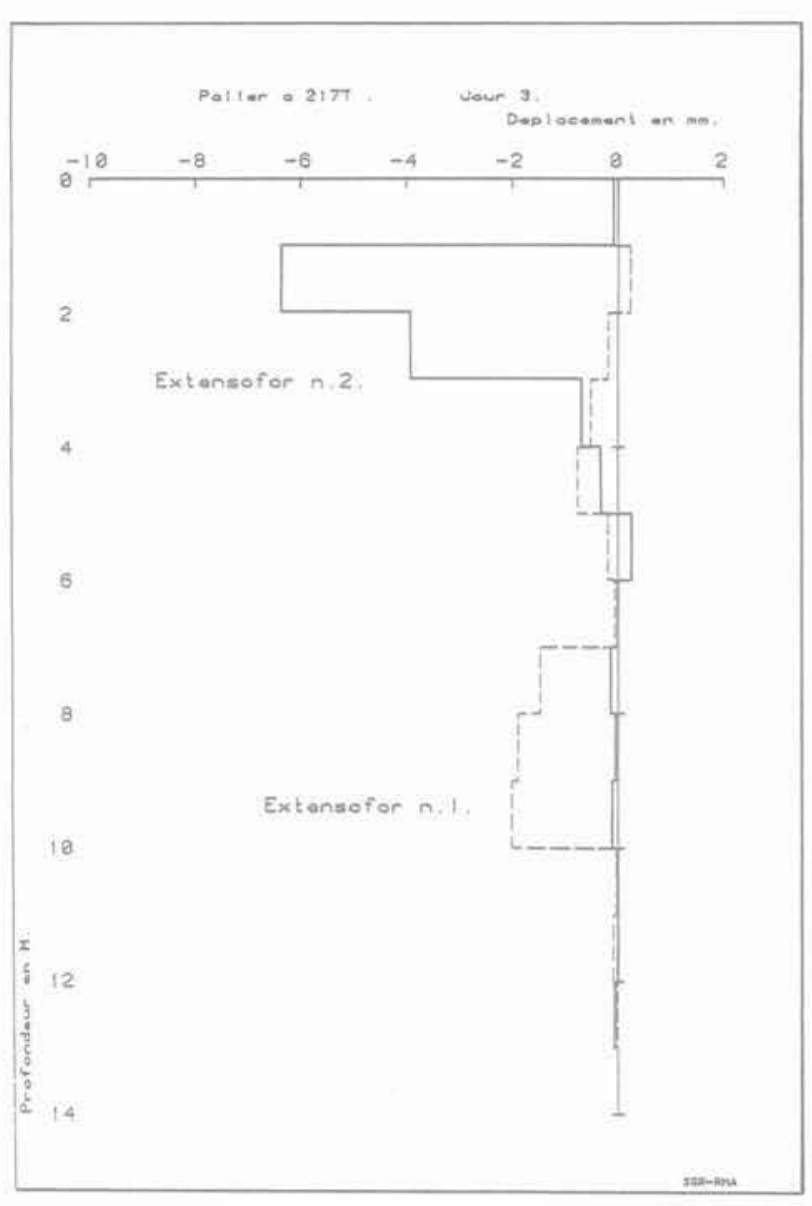

Fig. 11, - Semelle d'essai des Neyrolles.

normés constitué par le plan de la semelle et sa normale:

- le déplacement tangentiel (glissement) en fonction de la composante tangentielle de l'effort,

- le déplacement normal (poinçonnement) en fonction de la composante normale de l'effort.

et enfin:

- le déplacement vertical en fonction de la composante verticale de l'effort, courbe qui avait été obtenue très précocement sur le site gráce à l'utilisation du programme de traitement et du micro-ordinateur disponible sur le chantier (fig. 12).

L'examen de ces courbes ne permet pas de tirer de conclusions formelles quant à la mise en plasticitê. Il semble toutefois qu'au-delà de 8 à $10 \mathrm{MN}$ on aborde le domaine de mise en plasicité.

\subsubsection{Examen du fluage}

Pour essayer d'en juger, on a eu recours à l'examen des courbes de fluage sur les paliers pour lesquels on disposait des périodes de mesures assez longues:

jour 2: palier à $1,00 \mathrm{MN}(\mathrm{x} 4)$ avec tirants inclinés à $2 \mathrm{MN}$

jour 2: palier à $1,50 \mathrm{MN}(\mathrm{x} 4)$ avec tirants inclinés à $2 \mathrm{MN}$ jour 3: palier à $1,83 \mathrm{MN}(\mathrm{x} 4)$ avec tirants inclinés à $2 \mathrm{MN}$

jour 3: palier à 2,17 $\mathrm{MN}(\mathrm{x} 4)$ avec tirants inclinés à $2 \mathrm{MN}$

jour 4: palier à $1,50 \mathrm{MN}(\mathrm{x} 4)$ avec tirants inclinés détendus

jour 4: palier à $2,33 \mathrm{MN}(\mathrm{x} 4)$ avec tirants inclinés détendus

jour 4: palier à 2,50 $\mathrm{MN}(\mathrm{x} 4)$ avec tirants inclinés détendus

Les courbes de déplacement total correspondantes ont été tracées.

On a tenté de les exploiter suivant une procédure analogue à celle habituellement mise en cuvre pour les essais de tirants mais sans qu'il soit possible de mettre en évidence une limite de comportement élastique.

\subsection{Répartition des déplacements en profondeur}

\subsubsection{Mesures extensométriques}

On rappelle que les mesures extensométriques permettent de juger de la répartition des déformations le long d'un forage par l'appréciation du raccourcissement ou de l'allongement de base de mesures de longueur initiale $1,50 \mathrm{~m}$ matérialisées par deux bagues métalliques, la précision annoncée par le constructeur étant de $2 / 100 \mathrm{~mm}$.

a. Observations au jour $n^{\circ} 1$

- aucun mouvement au-delã de 10 mètres dans la zone d'ancrage;

- un raccourcissement de l'ordre de 1,5 à $2 \%$ entre 7 et 10 mètres dans la zone située immédiatement au-dessus;

- une zone neutralisée entre 5 et 7 mètres;

- un raccourcissement de l'ordre de 0,5 à $0,7 \%$ entre 3 et 5 mètres;

- entre 0.50 et $1 \mathrm{MN}$ on ne mesure pas de différence significative, sinon un allongement de $2 \mathrm{~mm}$ entre 5 et 7 mètres.

De telles observations ne sont pas faciles à interpréter. En effet: la déformation est maximale en profondeur, alors qu'avec une répartition des contraintes de type Boussinesq sous la semelle on pourrait s'attendre à l'inverse. C'est vraisemblablement lié à l'existence entre 7 et 10 métres d'une couche moins rigide, par exemple plus argileuse.

Globalement la déformation est un raccourcissement de l'ordre de 5 à $6 \mathrm{~mm}$.

b. au jour $n^{\circ} 2$, les tirants inclinés restant tendus, on observe la répartition des déformations sous $0,05,1,25$ et 1,50 MN; de charge verticale supplémentaire lou plus exactement $0,20,5$ et $6 \mathrm{MN}$ de charge verticale globale). 

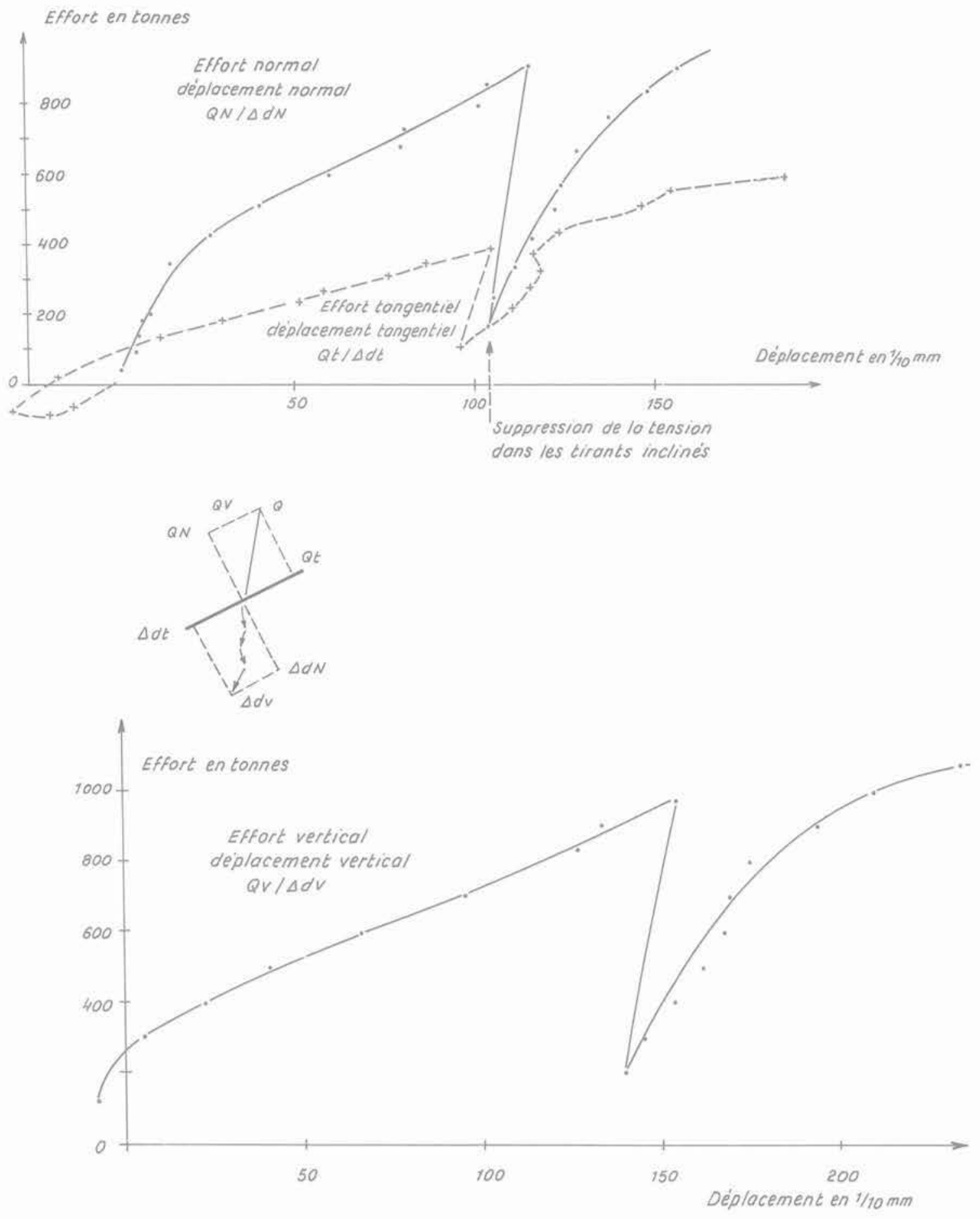

Fig. 12. - Décomposition du diagramme efforts/déformations. 
Le raccourcissement global est d'envrion $7,15 \mathrm{~mm}$, ce qui est assez conforme aux résultats de déplacement de surface.

c. Ultérieurement, cet Extensofor $n^{\circ} 1$ ne montre pratiquement plus aucune variation. Cela paraít normal puisque par rapport au déplacement global, il occupe une position radiale inclinée à $30^{\circ}$. Donc, au-delà de la phase de mise en tension initiale, il a été sollicité transversalement sous raccourcissement radial.

Il faut noter qu'il ne montre aucune restitution de déformation élastique lorsque les tirants inclinés sont détendus.

\subsubsection{Extensomètre $n^{\circ} 2$ vertical}

Pour cet extensomètre vertical, on retrouve une répartition des déformations conforme à Boussinesq avec, pour la charge à $6 \mathrm{MN}$ au $2^{e}$ jour:

- $4 \mathrm{~mm}$ de déformation entre 1 et 2 mètres,

$-2,5 \mathrm{~mm}$ de déformation entre 2 et 3 mètres.

Ces déformations allant en croissant pour atteindre au total 13 à $15 \mathrm{~mm}$ au $4^{e}$ jour.

En composant les déformations mesurées sur les deux extensomètres, on arrive à une très bonne corrélation avec les déformations mesurées en surface (même valeur de la composante normale au massif, valeur de la composante tangentielle mal mesurée par $\mathrm{E}_{1}$ ).

\subsubsection{Mesures inclinométriques}

Deux tubes inclinométriques avaient été mis en place. Aucune déformation dans le sens longitudinal $(\Delta x)$ n'a été mesurée dans les tubes. Quelques réajustements latéraux ont été perceptibles sur les deux tubes.

Une telle constatation est tout à fait logique, les déplacements mesurés en tête demeurant dans la limite de précision des mesures inclinométriques et surtout en l'absence de discontinuité de déplacement en profondeur significatif de l'existence d'une surface de rupture,

\subsection{Interprétation}

L'expérimentation menée sur la semelle des Neyrolles a permis de mettre en évidence certaines particularités du comportement d'une masse d'éboulis dont les caractéristiques physiques et géologiques avaient été testées par ailleurs grâce à l'exécution de puits.

Sous l'ensemble des sollicitations apportées, effort vertical avec ancrage ou effort vertical seul, les déformations sont restées faibles, de l'ordre de grandeur de ce que laissaient prévoir les modules pressiométriques. Leur composante majeure est verticale avec toutefois, lorsque l'ancrage incliné à $30^{\circ}$ est supprimé, une tendance au glissement suivant la pente qui s'accentue au-delà de $10 \mathrm{MN}$ de charge verticale.

On doit se demander ce qui se serait passé si la charge verticale seule avait été apportée sans que le terrain ne soit préalablement serré par la mise en tension des tirants inclinés. Il est vraisemblable que la composante tangentielle de la déformation aurait été plus forte, allant éventuellement jusqu'à un glissement superficiel.

Lors de la détente des tirants inclinés, la déformation enregistrée sur l'extensomètre $\mathrm{n}^{\circ} 1$ est restée irréversible. Il n'est pas interdit de penser que, compte tenu des déformations enregistrées dans le massif, une tension résiduelle soit restée dans les tirants sollicités passivement, ce qui tempérerait les conclusions optimistes que l'on peut être tenté de tirer du comportement sous charge verticale seule.

Les mesures inclinométriques n'ont montré aucune trace de cisaillement profond. Une approche de la stabilité par une méthode de Bishop et à deux dimensions montre que, pour de petits cercles de glissement (et il ne peut s'en développer de très grands, compte tenu de la présence du rocher en profondeur), le coefficient de sécurité reste supérieur à 1 dès que $\varnothing$ est supérieur à $35^{\circ}$. Pour la semelle en vraie grandeur, le rayon de «petit cercle» s'agrandit proportionnellement à la surface de la semelle et ce résultat prend beaucoup d'importance.

Enfin, les essais de fluage n'ont pas montré de tendance alarmante dans le domaine d'effort expérimenté.

Toutes ces constatations permettent de penser qu'une solution de fondation superficielle n'est pas à rejeter définitivement pour un ouvrage du type de celui qui était prévu ici, sous réserve toutefois:

- que de légers tassements différentiels soient admissibles:

- que puisse être assurée la pérennité à long terme de l'équilibre obtenu ici.

\section{CONCLUSION}

Sur le site d'une pile, les tassements d'une fondation sur semelle sont donc acceptables pour une charge égale à deux fois celle de l'ouvrage.

Mais l'incertitude sur le comportement mécanique des éboulis reste entière. Avec les caractéristiques prises en compte pour le projet, des calculs de stabilité générale à la rupture ont montré que l'influence des charges de louvrage est faible. Mais l'état de stabilité du versant est tout de même précaire. On ne peut pas se contenter des mêmes coefficients de sécurité pour louvrage. Un dispositif de confortement supplémentaire doit alors être prévu dans la zone de chaque fondation, par exemple longrines avec tirants d'ancrage (fig. 7).

Pour une fondation sur semelle correspondant à l'appui de la pile P 22 du viaduc de Neyrolles (comprenant une vingtaine de fondations similaires), un effort de confortement subhorizontal de $13 \mathrm{MN}$ serait nécessaire, correspondant à quatorze tirants de $0,90 \mathrm{MN}$.

D'autre part, vis-à-vis des tassements, se pose la 
question de la représentativité du site d'essai pour les autres appuis. On ne connaît pas le comportement au tassement d'éboulis de faible densité à indice des vides important, mais dont les grains de dimension importante peuvent s'arc-bouter les uns contre les autres. De toute façon, il y a toujours un risque de tassements différentiels dâ à la présence d'éboulis plus argileux. Dans ces conditions, il faudrait envisager pour chaque semelle la mise en place de tirants verticaux afin de réaliser un préchargement du sol avant construction de la pile et du tablier.

Toutes ces précautions, nécessitées pour une fondation sur semelle, conduisent à une dépense moyenne de 500000 francs en tirants d'ancrage par appui. Une telle somme permet de réaliser un puits de fondation de $4,5 \mathrm{~m}$ de diamètre et $19 \mathrm{~m}$ de profondeur. Pour l'ouvrage de Neyrolles la solution classique sur puits, à base éventuellement élargie, apparaît donc plus économique.
De la même manière, les essais de chargement de micropieux (unique et groupe de 3 ), ont donné d'excellents résultats. Il est possible de réaliser sans problème des micropieux de charge admissible 0,40 à $0,60 \mathrm{MN}$. Si on laisse de côté la résolution du problème de l'influence d'une telle fondation sur la stabilité générale (les micropieux travaillent surtout au frottement latéral et chargent les couches superficielles du versant), le cout d'une telle solution est également prohibitif: 30000 francs par mètre linéaire de profondeur contre 26600 francs pour un puits unique de $4.5 \mathrm{~m}$ de diamètre.

Il apparait donc que les solutions associant mieux les terrains de couverture aux fondations ne sont pas économiques par rapport aux solutions traditionnelles. Néanmoins, elles pourraient le devenir avec une meilleure connaissance du comportement mécanique des éboulis (surtout si le substratum est situé à une profondeur supérieure à $25 \mathrm{~m}$ ). 
